\title{
Mars Blue Clearing and Allais Effect
}

\author{
Russell Bagdoo \\ Saint-Bruno-de-Montarville, Quebec, Canada \\ Email: rbagdoo@gmail.com, Russellbagdoo@outlook.com
}

How to cite this paper: Bagdoo, R. (2022) Mars Blue Clearing and Allais Effect. Journal of Modern Physics, 13, 100-112. https://doi.org/10.4236/jmp.2022.132008

Received: January 5, 2022

Accepted: February 11, 2022

Published: February 14, 2022

Copyright (c) 2022 by author(s) and Scientific Research Publishing Inc. This work is licensed under the Creative Commons Attribution International License (CC BY 4.0).

http://creativecommons.org/licenses/by/4.0/

\begin{abstract}
Starting from the so-called "blue clearing" phenomenon, this paper establishes a link between disturbances of the Martian gravitational potential, the Allais effect of syzygy, astral influences and the Raman Stokes effect. This phenomenon is apparently peculiar to the Martian atmosphere. Photographs of Mars taken in blue light normally show only the atmosphere itself and clouds high above the surface. On occasion of oppositions, however, blue photographs will penetrate in varying degrees to the surface of Mars. Curiously, a burst of brightness and storms then occur on Mars. The atmosphere and clouds can be seen and photographed at short wavelengths by Earthbased telescopes equipped with a Wratten 47 filter. It happens that the blue screen of the filter suddenly begins to disappear and that the Martian surface becomes visible. The exact mechanism that produces blue clearing when Earth is between the Sun and Mars is highly speculative. We believe that the "Allais syzygy effect" may explain this phenomenon. The opposition would generate a "gravito-electromagnetic tension", which would spawn fluctuations in the gravitational potential of Mars, accompanied and linked to an electromagnetic effect. The outcome would be to trigger dust storms and exacerbate a disorderly excitement of molecules in the atmosphere. The thermal agitation facilitates the absorption of energy and the formation of small condensations that cause light scattering. Assuming that the Martian gravity decreased slightly, a Stokes Raman scattering would manifest at intramolecular level of the Martian atmosphere: the emitted photon has a lower energy than the absorbed photon. Therefore, it is mainly the waves corresponding to the spectral regions yellow, orange or red that are diffused, what eliminates short wavelengths. We deduce that the size of the inhomogeneities resulting from thermal excitation turns out to be greater than the length of the light waves of blue or purple regions of the spectrum.
\end{abstract}

\section{Keywords}

Blue Clearing, Allais Syzygy Effect, Astral Influences, Gravito-Electromagnetic Tension, Theory of Relation, Stokes Raman Effect 


\section{Introduction}

Mars, the first outer planet, possesses an orbit bigger than that of the Earth. Every two years, the celestial body becomes very brilliant and its apparent diameter is then widely up to 10 arcseconds, and may even reach the exceptional value of 25 arcseconds. This blaze in brightness occurs when Mars is in opposition, i.e. when the Earth is between the Sun and Mars. The opposition is the period when Mars is closest to Earth, which explains the high brightness of the planet and its high apparent diameter. At perihelion, the opposition occurs at a distance of 56 million kilometers from Earth, against 102 million kilometers at aphelion. Between an opposition in aphelion and in perihelion, the apparent diameter of Mars is halved. Because of the eccentricity of its orbit, and to a lesser extent, of the one of the Earth, the transition at the smallest distance can effectively take place up to 8 and a half days before or after opposition. The perihelic oppositions, which are most favorable for observation, are rare and only reproduce every 15 to 17 years on average. Mars, in opposition at perihelion, is 3.25 times as bright as when it is in opposition at aphelion [1] [2].

At aphelion, the distance from Mars to the Sun is 249 million kilometers, while it is only 207 million kilometers at perihelion. Therefore, the interval between two oppositions is not exactly 780 days, it is 810 days between two neighboring oppositions of the perihelion and 764 days for two neighboring oppositions of the aphelion. In the vicinity of an opposition, the celestial body began his retrograde motion. When Earth and Mars are diametrically opposed with regard to the sun, the distance between them may exceed the 400 million kilometers. The red planet is then easily confused with a star. Dust storms tend to develop precisely at perihelic opposition. The transparent state of the Martian atmosphere plays at least a role as important in the observation of the size of the disc; the dust in suspension may obscure many details of the surface. Although the size of the Martian disc reaches peaks during these oppositions, dust storms can degrade the viewing conditions, the surface of Mars appears then faded and blurred.

In addition to the spectacular dust storms, the generally clear layer of gases allows the observation of the clouds which sometimes stand out in a way contrasted on the Martian disc, and this in spite of their delicacy and their vaporous nature. If they are enough brilliant, these clouds can compete with the polar cap, sometimes becoming a source of confusion. Filters are found almost indispensable to differentiate atmospheric phenomena. Blue filter (W38A or W80) or purple (W47) are recommended to study them. The surface of Mars has a tendency to fade in favor of the thin atmosphere in this part of the spectrum. An altitude cloud will reach its maximum brightness with a blue filter, and will appear less prominent with a green filter (W58) or orange (W21). In contrast, while green or blue-green filters (W64) can bring out the mist, the ice surface and ice associated with the polar cap, and while the orange filter (W23A) or the red filter (W25) make appear more brilliant the deserts and distinguish the storms of dust, 
we notice that these phenomena are mitigated with a blue filter [3] [4].

\section{Martian blue clearing}

A notable exception, however, occurs when the "blue clearing" happens, a poorly understood phenomenon that American specialists have dubbed in this way and which surprised all those who saw it. From time to time, when Mars is in opposition with the Earth (Earth relatively to Mars occupies the same position as the Moon compared to Earth during a solar eclipse) every 2 years and 50 days on an average, the blue screen that hides all the details of the planet suddenly begins to disappear. The clearing can last for periods of several days, may be limited to one hemisphere and can vary in intensity from 0 (no surface features detected) to 3 (surface features can be seen as well as in the white light) [5].

The albedo of Mars is at best very feeble and, at worst, invisible through a blue filter, and the atmosphere and white clouds appear much brighter. The albedo appears vague through light blue filters, such as the Wratten 80A. With a dark blue filter (W47) or violet (380 - $420 \mathrm{~nm}$ ), the disc usually appears featureless except for clouds, hazes, and the polar regions [6]. The anomaly becomes an occasion where the markings on the surface of Mars can be seen clearly and photographed in the blue and violet light by Earth-based telescopes equipped with a Wratten 47 filter which is the standard for studying a blue clearing. Nobody seems to know why the Martian surface then becomes visible, why the atmosphere turns transparent into the blue and violet wavelengths.

\section{Overview of the first variations of the atmospheric transparency of Mars}

The mystery has a Martian history accompanied by a photographic progression that dates back to the beginning of the twentieth century. The author pays homage to the paper by Martz, Jr. E.P [7] which is the source of the previous related papers [8]-[20]. Researchers G. A. Tikhov, Pluvinel and Baldet pointed out the difference between photographs of Mars at long and short wavelengths, due to the darkening properties of the Martian atmosphere. W.H. Wright [8] has demonstrated it in detail. E. C. Slipher [9] first specifically noted the marked variation in transparency of the Martian atmosphere, to light of short wavelengths, at certain times. W. H. Pickering [10] also noted variation in the obscuring properties of the Martian atmosphere, on his early photographs of the planet. S. L. Hess [11] has pointed out that such variations in atmospheric transparency may have a very interesting effect on the color of the dark green surface markings. In 1941 he found a positive correlation between atmospheric transparency to blue light, and halting of the normal seasonal color changes, and an uncertain relationship in 1939, due to lack of a completely continuous series of photographs. R. S. Richardson [12] has discussed the general problem of atmospheric transparency on Mars, and the proposal of Hess, in an interesting popular article. G. P. Kuiper [13] discusses in some detail the problem of the Martian atmospheric haze and the seasonal variations of color as related to the nature of the surface markings.

G. de Vaucouleurs [14] reviews previously available observational material in regard to the atmospheric transparency on Mars and discusses the possible ori- 
gin of the obscuring medium. He points out that the variable obscuration can hardly arise from the variation in the molecular diffusion of the gaseous atmosphere and also that it is not likely to be due to variation in water droplets, surface dust, volcanic dust, or meteoritic dust suspended in the Martian atmosphere. S. L. Hess [15] has suggested that the variation of the blue haze may be due to forming and evaporation of high altitude clouds of frozen carbon dioxide crystals. G. P. Kuiper [13] indicates that the blue haze may be more likely due to frozen water crystals at somewhat lower levels. It has also been proposed that the blue haze may be due to day lit auroral emission in the upper Martian atmosphere.

During 1937 and 1939 E.P. Martz, Jr. [16] conducted extensive programs of photography of Mars (and other planets), by red (6500 A.U.), yellow (5600 A.U.), green (5300 A.U.), and blue-violet (4400 A.U.) light, from Mount Wilson, Griffith, and Steward Observatories. N.N. Sytinskaya [17] has discussed the variation in atmospheric turbidity across the disk of Mars, at the equator, for several different dates in 1939. E. C. Slipher [18] has noted that small variations in atmospheric transparency on Mars, to short wavelengths, occur from time to time, but that the major increases in transparency occur nearly coincidentally with opposition date of the planet. R. Wildt [19], E.P. Martz, Jr. [20] and others have suggested that the blueviolet Martian atmospheric haze may arise from variation in daylit-type auroral or fluorescence phenomena, rather than to particle scattering. Rosen has suggested that the blue-violet haze may arise from carbonsmoke type particles.

As can be seen, numerous hypotheses have been suggested as explanation of the phenomenon, like a "blue haze" on Mars concealing the surface at blue wavelengths. All such hypotheses have been found untenable until 1972 when a new explanation was offered simultaneously in the USSR and the U.S [21] [22]. It has been suggested that dust clouds may form above light regions because of an increase in the general circulation of the Martian atmosphere. This suggestion was confirmed in 1975 and led to a link between the appearance of these clouds and the intensity of blue clearing.

\section{Allais eclipse effect and Allais syzygy effect}

However, even with this explanation, the phenomenon remains poorly understood and the mystery remains unsolved. We consider that the anomaly of the blue clearing is similar to the anomalous movements exhibited by Maurice Allais pendulum at the time of a solar eclipse [23] and anticipate that a relationship must exist between the anomaly, the development of dusty clouds and the Allais effect [24].

Motion disturbances of a pendulum during a solar eclipse were observed for the first time by chance by Maurice Allais June 30, 1954, during measures of the azimuth of the plan of oscillation of a paraconical pendulum [24]. He observed a similar disturbance in 1959. This sudden change of the speed of precession of the plan of oscillation of the pendulum during an eclipse constitutes the Allais effect, also known as "Allais eclipse effect". He made two non-stop experiments during 
two periods of many planetary alignments, from 20 Nov. to 15 Dec. 1959 and from 15 March to 15 April 1960. In both experiments the azimuth had increased. On the other hand, Saxl and Allen (1970) observed with a torsion pendulum that these anomalies also applied to syzygies [25]. The latter are the phases of the lunar movement which find the Earth, the Moon and the Sun almost on the same line, that is to say, full Moon and new Moon. They bring the maximal height of tides. In 2002 and 2003, D. Olenici et S.B. Olenici, following the methodology of Maurice Allais, performed two non-stop experiments during periods of many planetary alignments [26]. All these experiments confirmed that the Allais effect appears during syzygies as well as during solar eclipses. An alignment without eclipse with a minimum of three celestial bodies is called a syzygy. The anomaly during alignment without eclipse with a minimum of three celestial bodies can be termed Allais syzygy effect [27]. The Allais effect becomes a general term that encompasses the Allais eclipse effect and the Allais syzygy effect (conjunction and opposition). The Allais eclipse effect is a special case of the Allais syzygy effect. We assume that when the Earth is between the Sun and Mars, the Allais effect (including syzygy and eclipse) causes fluctuations in the gravitational potential of Mars, what would be at the origin of the storms of dust and the blue clearing.

In the next discussion, we provide some precisions on the astral influences and the Allais syzygy effect. It is justified to the extent that it manages to establish on experimental bases the correspondences between celestial bodies and the Earth and makes touch of the finger the scientific reality. The last section takes us back to the hypothesis of the Allais syzygy effect: the effect would cause fluctuations in the gravitational potential of Mars, what would explain the blue clearing seen from Earth and the Stokes Raman effect of Martian atmosphere.

\section{Discussion: Allais Syzygy Effect and Astral influences}

If the hypothesis is correct, it depends on what is called "the phenomenon of astral influences". This phenomenon reveals the influences of the Sun on Earth and planets between them. It seems certain that they exist even if they are more complicated and confusing than imagined before. Our gravitational theories do not take these influences into account, as if the bottom of things had escaped us. To begin, let us say that in 1954, Einstein and the majority of the astronomers were opposed to the idea that the space was crossed by magnetic fields, that the Sun and the planets had an electric charge and that the electromagnetism could play a role in the celestial mechanics. In 1950, Immanuel Velikovsky, a psychoanalyst doctor, published a book in which he asserted that the space is not "empty" and that electromagnetism plays a fundamental role in the solar system. In early 1955, astronomers got radio signals from Jupiter. When Einstein, a few days before its death, learnt the news he used his influence to ensure that we experimentally verify the theories of Velikovsky [28] [29] [30]. From 1951, one had already noticed that the quality of the radio reception depends among others on the solar activity while leaving an unexplained residue. John $\mathrm{H}$. Nelson, pro- 
grammation analyst at R.C.A. Communications thought that this residue could be explained by the heliocentric position taken by the planets, i.e. with respect to the Sun. According to him, some very particular planetary configurations would disrupt the reception of radio waves: the days when the planets appear, relative to the sun, either at right angle to each other, either in conjunction or in opposition [31] [32]. In 1963, JA Roberts wrote an article in Planetary Space Science Research, showing that Venus, Jupiter and Saturn are the source of powerful radio wave emissions that the Earth is able to capture [33]. Since, artificial satellites have brought a revolution in the designs that one had of empty space and it has been shown that all the planets in the solar system have an electromagnetic field, even if those of Mercury, Mars and Venus are weak [34] [35].

Therefore, we can consider that the planets are giant electromagnets that describe with prodigious speeds their revolutions around the Sun, the central electromagnet. The Sun around which they describe their orbits, has a considerable static electricity charge to give rise an "electrostatic field" around the celestial bodies of the solar system. As in general, the core of each of the planets is quite a good electrical conductor, this core behaves, by moving in the magnetic field, as the induced of a dynamoelectric machine or of a magneto turning between poles of its magnet. It follows that, according to the classical laws of electromagnetic induction, any cause which will make vary the intensity of the field, or any cause which will make vary the speed of displacement of the conductive body in the field, will modify the intensity of the observed effects [36] [37] [38].

The theory of general relativity is accountable to both the nature of matter and motion of stars by hanging on to the experimental reality. Matter a curvature of space creates gravity. However, the gravitational field, framed with precision in the network of its formulas, lets escape the energy, or the electromagnetic radiation [39]. The Cosmos is made of matter and energy, and the solar system is no exception. Matter evokes inertia while radiant energy is active. Universal gravitation causes the bodies to exert an attraction on each other. In our solar universe, the radiant energy originally engendered the matter. This one became a reservoir of forces and movements. Celestial bodies, grouped into overlapped entities, act by gravitation on each other and maintain their respective movements. In turn these movements facilitate and diversify transformations that radiant energy is likely to operate on all of them. Viewed in isolation, stars acquire their own dynamics that have double character of gravity and electromagnetism [40] [41] [42]. Although relativity gives a picture of the physical universe while being unable to account for the energy, we can remark that the curvature of the light of the theory concerns the electromagnetism in connection with a gravitational potential. This is not a purely gravitational effect like for the advance of the perihelion of Mercury or the gravitational redshift [43]. We also note that the great majority of the gravitational systems which we meet in the physical universe are systems in constant total mass. In this context, according to the general relativity, the electromagnetism goes hand in hand with the gravitational: light is bent in proportion by matter (mass); more the gravitational po- 
tential is big, more the light is bending.

Nevertheless, when there are disturbances of move of gravitational measurement instruments that raise or lower the gravitational potential, contemporary physicists have come to deny the facts to dodge the discomfort of these mysterious disruptions that do not fit the established theories. It is nevertheless necessary to become aware that if these disturbances are connected to physical phenomena observed during the syzygies and the solar eclipses, and if they are applied on both the mass and the light, we have to involve gravitation and electromagnetism in the fundamental principle of a "gravito-electromagnetic dynamics".

Let us remind that disturbances of movement of a pendulum during a solar eclipse were observed for the first time in a fortuitous way by Maurice Allais on June 30th, 1954, during measures of the azimuth of the plane of oscillation of a paraconical pendulum [24]. This sudden change of the speed of precession of the plane of oscillation of the pendulum during an eclipse constitutes the Allais effect, also called "Allais eclipse effect". On the other hand, Saxl and Allen observed with a torsion pendulum that these anomalies could also be applied to the syzygies [25]. The latter are the phases of the lunar movement which find again Earth, Moon and Sun more or less on the same line, that is to say Full moon and New Moon. They bring back the maximum height of the tides. An alignment without eclipse with a minimum of three celestial bodies can be called Allais syzygy effect. The experiments of Maurice Allais of November-December, 1959 and March-April, 1960 with the paraconical pendulum confirmed the existence of inexplicable periodic structures within the framework of Newtonian mechanics and relativistic mechanics, amplitudes hundred million times larger than the amplitudes calculated with the current theory. They also allowed to demonstrate the existence of a direction of variable anisotropy over time and to specify the azimuth at any time. M. Allais interprets this anisotropy of space as corresponding to an anisotropy of inertia according to the considered direction of the anisotropy of space and resulting of astronomical influences. These promising experiments were not able to continue due to the closure of his laboratory at SaintGermain on June 1960, following a cabal [24].

If anomalies of the paraconical pendulum must be highlighted during a configuration opposing the Sun and Mars, they would serve to demonstrate that the influence of the Sun and the Earth on Mars entails an anisotropic space, variable with time in direction and in intensity. And to determine if there is an abnormal "gravitational tension" which affects the gravitational potential and engenders the observed hurricanes.

Let us add that we were interested in the discoveries and deductions of $\mathrm{M}$. Allais because they were in agreement with the theory of the Relation [44] [45] [46]. According to this theory, under the principle of compensation, the electromagnetic space of inertia which, globally, decreases is offset by an increase of the gravific space. When we talk of inertia of space, it is an inertia that opposes gravity (like that of special relativity) and not of the inertial forces that accom- 
pany the gravitational forces in the same direction. Locally, within a gravific space which becomes suddenly non-static, the energy of an electromagnetic space of inertia augments, activates and is counterbalanced by a gravific space that abates. In the event of an eclipse or a syzygy which would disrupt momentarily the celestial body in the point to make slightly vary downward the reduction its gravitational potential, we shall say that the "electromagnetic" mass of the photon (we know that the photon has no energy of rest) the equation $m=h v / c^{2}$ increased and that the electromagnetic activity within the celestial body grew at the expense of the gravity. According to Einstein's equivalence principle, the photon has a "gravitational mass" equivalent to an "inertial mass" and equal to $h v / c^{2}$. According to the relation $v^{\prime}=v\left(1+g l / c^{2}\right)$, a photon emitted by Mars will have a frequency a little higher at his arrival at the Earth's surface, with mass $h v / c^{2}$, supposedly constant during the journey [47]. That this mass and also the "proper" time seem modified during the state of eclipse or syzygy, it is the proof that we are dealing with an anomaly similar to the Pioneer effect that makes vary the "invariance".

\section{Blue Clearing, Allais Syzygy Effect and Raman Stokes Effect}

\section{Blue clearing seen from Earth and Allais syzygy effect}

After these explanations which are other thing than the knowledge of appearances, we can return to our supposition: when the Earth is between the Sun and the Mars, the Allais effect causes fluctuations in the gravitational potential of Mars ( $\Phi=v^{2}=g l$ ), what would be at the origin of swirls of dust and of the blue clearing.

With a blue filter, the Earth perceives Mars with a light that corresponds to short waves in the blue region of the spectrum. During the blue clearing-i.e., the disappearance of the blue screen of the filter-the Earth sees Mars with a light that corresponds to longer wavelengths in the yellow, orange or red areas of the spectrum.

In fact, if the light collected through the blue filter of the telescopes on Earth was envisaged as radio signals sent by Mars, the interval between two signals would be longer, giving a slight redshift which can be interpreted as a slight decrease of the attraction of the Sun, or the Sun-Earth tandem. By Doppler effect, we could believe that Mars suddenly goes away, that there is a kind of redshift, as if the Earth, or rather the Sun behind, attracted less Mars. The observer on Earth has the impression that during the time $t=l / c$ Mars moves of the distance $I$ outwards with the acceleration $g$. This is effectively the inverse of the Pioneer anomaly [46]. In term of gravitational frequency shift, there is a tiny abnormal blueshift.

If we consider that in normal times the quantum of energy received by the Earth from Mars is $\varepsilon=h v$ and if we assume that the light is emitted from Mars at the distance $I$ (distance Mars-Earth) [48], the total energy of a photon of frequency $v$ and energy $h v$, reaching the earth's surface becomes 


$$
h v^{\prime}=h v+h v g l / c^{2} .
$$

The receiver located on the Earth's surface detects a frequency $v^{\prime}$ superior to $v$ from the Martian source ( $g$ is the Earth's gravitational field):

$$
v^{\prime}=v\left[1+g l / c^{2}\right] \text {. }
$$

We can say that during the blue clearing, Mars acts as if it was moving away of a distance $I$ from the Earth, because of a potential loss of attraction. The Earth's gravitational field $g$ amounted to $g-g^{\prime}$. When a photon emitted by Mars reaches the Earth's surface, it has lost the potential energy $\left(h v / c^{2}\right)\left(g-g^{\prime}\right) l$ and won the kinetic energy $\left(h v / c^{2}\right)\left(g-g^{\prime}\right) l$. Its total energy has become

$$
h\left(v^{\prime}-v^{\prime \prime}\right)=h v+\left(h v / c^{2}\right)\left(g-g^{\prime}\right) l .
$$

The frequency $v^{\prime}-v^{\prime \prime}$ of the photon at his arrival at the Earth's surface is less red-shifted relative to its initial frequency according to the relation

$$
\left(v^{\prime}-v^{\prime \prime}\right)=v\left[1+\left(g-g^{\prime}\right) l / c^{2}\right] \text {. }
$$

During the phenomenon, the receiver disposed on the Earth's ground, detects a frequency $v^{\prime}-v^{\prime \prime}$ slightly smaller than $v^{\prime}$ without the blue clearing. This means a small blueshift for Mars.

\section{Allais effect and Stokes Raman effect of the atmosphere of Mars}

We assume that the gravitational potential of Mars varies when the Earth is between the Sun and Mars, what would have the effect of triggering dust storms, heavy atmospheric variations and cloud formations, which are related to the time of these oppositions. This change would exacerbate a disorderly animation of molecules in the atmosphere. The thermal agitation would encourage creation of tiny rarefactions or condensations and it is them that cause the light scattering, because they disturb the optical homogeneity of the atmosphere. The optical medium becomes heterogeneous and the incident light is scattered laterally.

Atom or molecule stores energy in their excited state. A molecule can be excited to a very high energy state. The amount of energy necessary to reach this state is $h v_{o}$. That energy is released when the molecule returns to a lower state. The return to the ground-state vibrational energy level $v=0$ results in the emission of a light that has the frequency $v_{o}$. This emission is usually observed in the visible spectral region and is called Rayleigh scattering. Nevertheless, we think that what is happening to the intramolecular level of the atoms, that constitute the molecules of the Martian atmosphere which diffuses waves, can be likened to a Stokes Raman effect [49] [50].

The scattering of light on the optical modes is called Raman scattering. It is different from the Rayleigh scattering because the scattered light changes the frequency of the spectrum active vibration. Historically, the effect was first observed with molecules. Molecules vibrate, and each molecular vibration corresponds to a certain amount of energy. In the scattering process, this energy is added or subtracted from the incident light. A Stokes Raman effect occurs when the molecule absorbs the incident light of frequency $v_{o}$ and reemits light at a 
lower frequency.

Thus, during the time of opposition and blue clearing, the gravitational potential $\Phi$ of Mars would be perturbed. The molecule would then be relaxed from the excited state and would not completely relaxed at the level of energy minimal, $v=0$, but would stops at $v=1$, or even at an upper energy level. The energy of the emitted photon would be lower, we would have $E_{S}=h\left(v_{o}-v_{1}\right)$, where $S$ stands for Stokes. The energy emitted in this process would be decreased of $h v_{1}$. Spectral lines with frequencies smaller than $v_{o}$ are called Stokes lines [51] [52] [53].

In this way, if the Martian gravity varies in decreasing a bit, the tiny rarefactions lose in condensation, enlarge and the dimensions of the inhomogeneities resulting from the thermal excitation are proving to be greater than the length of light waves. From then on, it is mainly the waves corresponding to the yellow, orange or red regions of the spectrum which are diffused, what would have consequence to rule out the waves corresponding to purple or blue regions of the spectrum [54].

\section{Conclusions}

This paper establishes for the first time a link between the phenomenon of "blue clearing", violent storms and the Allais effect of syzygy. American specialists have given the name of blue clearing to an upheaval that violently surprised them: when Mars is in opposition (Earth between Mars and Sun), the blue screen that hides the details of the planet in some filters, suddenly begins to disappear, during a few days. However, some confusion seems to surround the term "blue clearing of Mars". The tumult has nothing to do with strong dust storms giving way to a calm clarity or to an unexpected illumination of the planet atmosphere. It simply eliminates the blue color of the filter of an observation instrument placed on Earth or in space.

At the end of the Introduction we argued that the blue clearing is caused in the first instance by the Allais syzygy effect. We see a close relationship between the episode of Mars blue clearing and the eclipse effect during solar eclipses observed by Maurice Allais. In the first case, we observe on the blue filter a brutal change of wavelength; the filter becomes fortuitously a sort of measure of the wavelength or frequency. In the second case, the paraconical pendulum is an instrument to study certain comportments of Earth's gravity. If anomalies of the paraconical pendulum must be highlighted during a configuration opposing the Sun and Mars, they would serve to demonstrate that the influence of the Sun and the Earth on Mars entails an anisotropic space, variable with time in direction and in intensity, and to determine if there is an abnormal "gravitational tension" which affects the gravitational potential and engenders the observed hurricanes.

The surface of Mars becomes visible when the blue color corresponding to a certain wavelength disappears from the blue filter which allows exclusive obser- 
vation of the Martian atmosphere. This frequency shift signifies an alteration of the gravitational potential of Mars. It often occurs during syzygy at the time of unusual strong storms which are symptomatic of disturbances within the planet and can affect its rotation on itself. Since these disturbances are exerted on both mass and light, the blue clearing phenomenon makes it possible to realize that we must involve gravity and electromagnetism in the fundamental principle of "gravito-electromagnetic dynamics".

In a broader way, during the Discussion, we glimpsed that if the hypothesis is correct, it falls under what is called "the phenomenon of astral influences" which reveals the influences of the Sun on the Earth and of the planets between them. It seems certain that these influences exist even if they are more complicated and confusing than previously imagined. Our gravitational theories do not take this into account. No spectral analysis of the "reflectors" that are the planets has never really been delivered, and no scientist has ever dared to involve universal gravitation and electromagnetism in an exhaustive study of the impulse of celestial bodies. Of course, a "gravitational tension" was noticed when the planets get closer or go away as well as an "electromagnetic tension" related to solar activity, but this paper is referring to a gravito-electromagnetic tension. The bodies of the solar system generate magnetic fields which intersect, constantly react by induction on one another, amending at each instant their vibratory modulations according to the displacements of their respective positions. In brief, there would be a physical influence of celestial bodies linked up with "gravitational and electromagnetic currents". Each planet would exert a specific action and there would be angular relationships between the planets or luminaries with each other and with the Earth.

In a more restricted way, we saw in the section "Blue clearing, Allais syzygy effect and Raman Stokes effect" that in the event of the case of the opposition, whereas Mars is induced by the Sun and the Earth, one would have an effect gravito-electromagnetic of syzygy: the energy of the vibrations of the molecules would be disturbed and modified. Thenceforward that the Martian gravity decreases slightly, we can conceive that the energy of the emitted photon can be weaker by Stokes Raman scattering and that the blue of the spectrum can give way to longer wavelengths, which would remove the blue filter screen and make all the details of the planet appear. If we bring clarifications on the physical meaning of the variation of the gravitational potential and on a Raman effect which involves a scattered light above or below the standard frequencies, it will be possible to better understand the influence of the celestial bodies with each other.

\section{Conflicts of Interest}

The author declares no conflicts of interest regarding the publication of this paper.

\section{References}

[1] Mars, Wikipedia. https://en.wikipedia.org/wiki/Mars 
[2] Labrot, L'observation de Mars (1997-2021). https://www.nirgal.net/observe.html

[3] Troiani, D.M. (2003 \& 2005) Mars at Its All-Time Finest, Sky \& Telescope. https://skyandtelescope.org/astronomy-news/observing-news/mars-at-its-all-time-fi nest

[4] Nakakushi, T., Akabane, T., Iwasaki, K. and Larson, S.M. (1999) The Blue Clearing at Syrtis Major and Its Relation to Equatorial Clouds: 1997 and 1999 Observations.

[5] James, P.B., Bell III, J.F., Clancy, R.T., et al. (1996) Journal of Geophysical Research, 101, 18883-18890. https://doi.org/10.1029/96JE01605

[6] The International MarsWatch Electronic Newsletter (1998) Volume 3; Issue 6.

[7] Martz Jr., E.P. (1954) Astronomical Society of the Pacific, 66, 45-51. https://doi.org/10.1086/126651

[8] Wright, W.H. (Oct. 1939) Publications of the Astronomical Society of the Pacific, 51, 292-293. https://doi.org/10.1086/125088

[9] Slipher, E.C. (June 1937) Publications of the Astronomical Society of the Pacific, 49, 137. https://doi.org/10.1086/124789

[10] Plotkin, H. (1993) Journal for the History of Astronomy, 24, 101-122. https://doi.org/10.1177/002182869302400104

[11] Hess, S.L. (1952) Final Report on the Study of Planetary Atmospheres (Lowell Obs., U.S. Air Force Contract). 42.

[12] Richardson, R.S. (1953) Astounding Science Fiction, 42, 117. https://doi.org/10.1126/science.117.3042.42.s

[13] Kuiper, G.P. (1952) Atmospheres of the Earth and Planets. 2nd Edition, Univ. of Chicago Press, Chicago, 394, 398.

[14] De Vaucouleurs, G. (1951) Physique de la Planète Mars. Michel, Paris, 28, 55, 114.

[15] Hess, S.L. (1950) Meteorology, 7, 8.

[16] Martz Jr., E.P. (1953) Astronomical Journal, 58, 221. https://doi.org/10.1086/106866

[17] Sytinskaya, N.N. (1964) Soviet Astronomy, 7, 541.

[18] Slipher, E.C. (1953, Oct. 22-23) Mars Committee Meeting, Lowell Obs.

[19] Wikipedia. Rupert Wildt. https://en.wikipedia.org/wiki/Rupert Wildt

[20] Martz Jr., E.P. (1939-47) Correspondence and Unpublished Report.

[21] Thompson, D.T. (Nov 1972) Reviews of Geophysics, 10, 919-933. https://doi.org/10.1029/RG010i004p00919

[22] Prokofeva, V.V., Chuprakova, T.A., Dziamko, S.S. and Bryzgalova, T.V. (1976) Soviet Astronomy, 19, 378-384.

[23] Bagdoo, R. (2009) Journal of Modern Physics, 11, 1620-1638. https://doi.org/10.4236/jmp.2020.1110101

[24] Allais, M. (1997) L'Anisotropie de l'Espace. Edition Clément Juglar. 50, 212, 255, $326,330$.

[25] Saxl, E.J. and Allen, M. (1971) Physical Review D, 3, 823-825. https://doi.org/10.1103/PhysRevD.3.823

[26] Popescu, V.A. and Olenici, D. (2006) A Confirmation of the Allais and JeverdanRusu-Antonescu Effects during the Solar Eclipse from 22 September 2006, and Quantization of Behaviour of Pendulum. Semantic Scholar. Corpus ID: 124079594 http://www.hessdalen.org/sse/program/Articol.pdf 
[27] Glotz, M. (1969) La gravité, Sciences du Monde. Librairie Jules Tallandier, Paris, 34-41.

[28] Velikovsky, I. (1950) Worlds in Collision. Paradigma Ltd. https://doi.org/10.1063/1.3066934 https://www.paradigma-publishing.com/

[29] Warshofsky, F. (1976) L'étonnante théorie de Velikovsky, Sélection du Reader's Digest. 180-194.

[30] Immanuel Velikovsky. https://en.wikipedia.org/wiki/Immanuel Velikovsky

[31] Nelson, J.H. (1951) R.C.A. Review, 12, 26.

[32] Gauquelin, M. (1970) Les horloges cosmiques. Denoël, Paris, 119-120.

[33] Roberts, J.A. (1963) Planet Space Science, 11, 221-232. https://doi.org/10.1016/0032-0633(63)90026-6

[34] Brann, T., Steigerwald, B. and Jones, N. (2020) MAVEN Maps Electric Currents around Mars. NASA Goddard Space Flight Center, Greenbelt.

[35] Magnetosphere, Wikipedia. https://en.wikipedia.org/wiki/Magnetosphere

[36] Hoyle, F. (1955) Frontiers of Astronomy. Signet Science Library Books, New York, 83-102.

[37] Morris, R. (1985) Times Arrows. Simon \& Schuster, New York, 135-148.

[38] Bagga, T. The Outer Planets; Magnetospheres. https://lasp.colorado.edu/outerplanets/giantplanets magnetospheres.php

[39] Rousseau, P. (1950) La conquête de la science. Librairie Arthème Fayard, Pairs, 316-320.

[40] Star, Wikipedia. https://en.wikipedia.org/wiki/Star

[41] Heckel, P. (1973) L'homme et les influences astrales. Epi s.a. Editeur, Paris, 17-18.

[42] Sackmann, I.J., Boothroyd, A.I. and Kraemer, K.E. (1993) Astrophysical Journal, 418, 457. https://doi.org/10.1086/173407

[43] Schrödinger, E. (1950) Space-Time Structure. Cambridge University Press, Cambridge, $1,2$.

[44] Bagdoo, R. (2019) Journal of Modern Physics, 10, 310-343. https://doi.org/10.4236/jmp.2019.103022

[45] Bagdoo, R. (2013) The Energy in Virtue of the Principle of Compensation.

[46] Bagdoo, R. (2020) Journal of Modern Physics, 11, 616-647. https://doi.org/10.4236/jmp.2020.115041

[47] Rocard, J.-M. (1986) Newton et la relativité, Que sais-je? Presses Universitaires de France, Paris, 102-103.

[48] Mavridès, S. (1988) La Relativité, Que sais-je? Presses Universitaires de France, Paris, 105-106, 112.

[49] Fateley, W.G. and Bentley, F.F. (1990) Raman Spectroscopy. In: Encyclopedia of Physics, VCH Publishers, Inc., Hoboken, 1034.

[50] Bagdoo, R. (2021) Journal of Modern Physics, 12, 1783-1794. https://doi.org/10.4236/jmp.2021.1213104

[51] Möller, K.D. (1988) Optics. University Science Books, Sausalito, 574-575, 621.

[52] Raman Scattering. https://en.wikipedia.org/wiki/Raman scattering

[53] Raman, C.V. (1928) Indian Journal of Physics, 2, 387-398.

[54] Radounskaïa, I. (1972) Idées folles. Édition MIR, Moscou, 78-82. https://doi.org/10.1111/j.1432-1033.1975.tb04034.x 1 